Gefässchirurgie 2011 16:330-331

DOI 10.1007/s00772-011-0939-6

๑) Springer-Verlag 2011

\author{
M. Augustin ${ }^{1,3} \cdot$ S. Debus ${ }^{2,3}$ \\ ${ }^{1}$ Institut für Versorgungsforschung in der Dematologie und bei \\ Pflegeberufen, Universitätsklinikum Hamburg-Eppendorf, Hamburg \\ 2 Klinik und Poliklinik für Gefäßmedizin, Universitäres Herzzentrum, \\ Universitätsklinikum Hamburg-Eppendorf, Hamburg \\ ${ }^{3}$ Comprehensive Wound Center (CWC), Competenzcentrum \\ Versorgungsforschung bei vaskulären Erkrankungen (CVvasc), Hamburg
}

\title{
Versorgungsforschung in der Gefäßmedizin
}

neiden, das aber zahlreichen Herausforderungen ausgesetzt ist.

Die Zukunft der Gefäßchirurgie wird sich dabei nicht nur um die kurzfristige Frage angemessener Finanzierungen drehen, sondern um die Bereitschaft und die Fähigkeit zur Modernisierung, Akademisierung und Selbstregulierung. widerspiegeln. Viele der modernen therapeutischen Verfahren waren noch vor 10 Jahren undenkbar. Man erinnere sich nur an die molekulare Diagnostik, die biotechnologischen Verfahren, den Siegeszug der endovaskulären Techniken, verbesserte medikamentöse Therapie oder die sich anbahnenden Potenziale der regenerativen Medizin. Die Gefäßmedizin erweist sich hier als zukunftsgerichtetes forschendes und innovierendes Fach mit enormem Entwicklungspotenzial. Dennoch der Spagat zwischen dem Potenzial moderner Technologien und der Ist-Situation unserer gefäßmedizinischen Versorgung ist eine große Herausforderung. Diese wird wesentlich von den ökonomischen und sozialgesetzlichen Rahmenbedingungen unseres Gesundheitssystems geprägt, das auf der einen Seite nach Innovationen ruft, auf der anderen Seite aber den Preis für medizinischen Fortschritt häufig nicht zu zahlen bereit ist. Vor diesem Hintergrund und den vielfältigen ökonomischen und gesundheitspolitischen Rahmenbedingungen ist eines klar geworden: Das Fach Gefäßchirurgie befindet sich in einer zentralen versorgenden Rolle, um die uns Kollegen vieler anderer Länder be-

\section{(> Die Gefäßmedizin ist ein zukunftsgerichtetes forschendes und innovierendes Fach mit enormem Entwicklungspotenzial}

Die dafür notwendigen Schritte werden in der Auseinandersetzung mit den Rahmenbedingungen unseres Gesundheitssystems entschieden. Ein wichtiger und wahrscheinlich strategisch entscheidender Faktor ist dabei die Hoheit über fundierte Daten zur Versorgung von Gefäßerkrankungen in Deutschland. Diese Datenhoheit bezieht sich auf die Epidemiologie, den Versorgungsbedarf, die Versorgungsqualität, auf die Kosten und den Nutzen der Versorgung bis hin zur gefäßchirurgischen Versorgungskompetenz. Die Deutsche Gesellschaft für Gefäßchirurgie und Gefäßmedizin hat mit der Einrichtung des Deutschen Instituts für Gesundheitsforschung in der Gefä $\beta$ medizin (DIGG) die Notwendigkeit einer koordinierten Versorgungsforschung erkannt und die Weichen für die langfristige Aufstellung der Versorgungsforschung gestellt. Diese wird seit 2011 durch die en- ge Zusammenarbeit mit dem Competenzzentrum für Versorgungsforschung bei vaskulären Erkrankungen (CVvasc) des Universitätsklinikum Hamburg-Eppendorf wahrgenommen. Mit Projekten zum Aortenscreening, zur Versorgungsqualität vaskulärer Wunden, zur Epidemiologie vaskulärer Wunden sowie zur vaskulären Morbidität der deutschen Bevölkerung wurde die Arbeit aufgenommen. Erste gesundheitsökonomische Daten wurden bereits publiziert [5]. Ziel ist es, mit diesen und weiteren Projekten Daten und Argumentationshilfen zur Verfügung zu stellen, mit deren Hilfe eine evidenzbasierte Diskussion mit der Gesundheitspolitik zur Versorgung vaskulärer Erkrankungen möglich wird.

Ein wesentliches Novum des letzten Jahrzehnts ist die Etablierung von Patientenregistern, die wichtige Daten aus der Routineversorgung in strukturierter, valider Form zusammenführen und somit eine Brücke schlagen zwischen der klinischen Forschung und der Versorgungsforschung. Patientenregister haben eine zentrale Bedeutung für eine versorgungsnahe Forschung, jedoch ist eine fundierte Methodik essenziell. Für diese wurde im Jahre 2010 mit dem Memorandum "Register in der Versorgungsforschung“ ein bundesweiter Konsens publiziert, der als Orientierungshilfe für die Versorgungsforschung dient [4]. Hier wird ferner die Differenzierung verdeutlicht, mit der Patientenregister zu betrachten sind. Die Unterscheidung zwischen Melde- und 
Therapieregistern sowie deren jeweiligen Varianten ist für die Forschung und für die Übertragung der Ergebnisse in die Praxis wesentlich. Die Versorgungsforschung bedarf in jeder Hinsicht einer methodisch fundierten, qualitativ hochwertigen Umsetzung. Die Rahmenbedingungen hierzu wurden auch in weiteren Methodenpapieren des Deutschen Netzwerkes für Versorgungsforschung erarbeitet, welche die Grundlage für die zukünftige Arbeit des DIGG und des CVvasc darstellen [3].

Wir stehen in der gefäßmedizinischen Versorgungsforschung in Deutschland am Anfang; andere Fachgesellschaften sind hier wesentlich weiter. Beispielsweise haben sich die deutschen Dermatologen über ihre Fachgesellschaften DDG und den Berufsverband BVDD als eine der ersten Fachrichtungen konsequent der Aufgabe einer Versorgungsforschung in Deutschland angenommen - mit beträchtlichem versorgungspolitischem Nutzen [1]. Die weitere Forschung und die Umsetzung der Ergebnisse aus der Versorgungsforschung werden in den nächsten Jahren eine herausragende Bedeutung bei der Positionierung der Gefäßmedizin im deutschen Gesundheitssektor haben. Sie werden insbesondere aber die Anliegen unserer Patienten nach einer qualifizierten und humanen Krankenversorgung unterstützen.

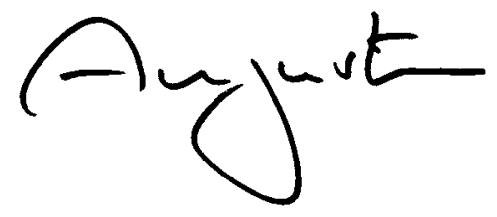

Prof. Dr. M. Augustin,

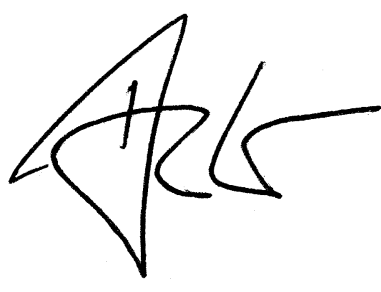

Prof. Dr. E. Sebastian Debus

\section{Korrespondenzadresse}

Prof. Dr. M. Augustin

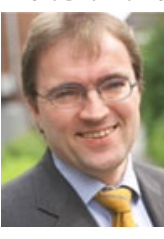

Institut für

Versorgungsforschung in der

Dematologie und bei

Pflegeberufen,

Universitätsklinikum

Hamburg-Eppendorf

Martinistr. 52, 20246 Hamburg

m.augustin@uke.

uni-hamburg.de

\section{Prof. Dr. S. Debus}

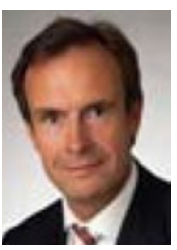

Klinik und Poliklinik für

Gefäßmedizin, Universitäres

Herzzentrum,

Universitätsklinikum

Hamburg-Eppendorf

Martinistr. 52, 20246 Hamburg

s.debus@uke.uni-hamburg.de

\section{Literatur}

1. Augustin M, Reich K, Reusch M et al (2009) Health services research in psoriasis - the German approach. Dermatology 218(4):293-301

2. Franzke N, Augustin M (2010) Dermatologie. In: Pfaff H, Neugebauer EA, Glaeske G, Schrappe M (Hrsg) Lehrbuch Versorgungsforschung. Systematik - Methodik - Anwendung. 1. Aufl. Schattauer

3. Glaeske G, Augustin M, Abholz H et al (2009) Epidemiologische Methoden für die Versorgungsforschung. Gesundheitswesen 71:685-693

4. Müller D, Augustin M, Banik N et al (2010) Memorandum Register für die Versorgungsforschung - Memorandum Registry for Health Services Research. Gesundheitswesen 72(11):824-839

5. Purwins S, Herberger K, Debus ES et al (2010) Costof-illness of chronic leg ulcers in Germany. Int Wound J 7:97-102

\section{Wie aktivierte Blutgefäßzellen} ruhiggestellt werden können

In einer aktuellen Publikation beschreiben Wissenschaftler, dass der Transkriptionsfaktor HOXC9, der in intakten, ruhenden Endothelzellen stark produziert wird, in aktivierten Endothelzellen des Blutgefäßsystems nicht mehr gebildet wird. Umgekehrt kann eine verstärkte „künstliche“ Bildung von HOXC9 aktivierte Endothelzellen in einen ruhenden, aber dennoch funktionellen Zustand versetzen.

Die Wissenschaftler konnten sowohl in kultivierten Endothelzellen als auch in Blutgefäßen im Zebrafisch zeigen, dass HOXC9 die Bildung des für Blutgefäße wichtigen Faktors Interleukin 8 hemmt und auf diese Weise eine Aktivierung der Endothelzellen und Neubildung der Blutgefäße verhindern kann. Dabei wiesen sie im Detail nach, dass HOXC9 vielfältige Prozesse der Blutgefäßbildung, wie die Neubildung und Wanderung von Endothelzellen hemmt, und dass diese Funktionen von HOXC9 ausschließlich über den Faktor Interleukin 8 vermittelt werden.

Im Mittelpunkt der weiteren Forschung in diesem Zusammenhang steht insbesondere die Frage, ob eine gesteigerte Bildung von HOXC9 nicht nur aktivierte Endothelzellen in einen Ruhezustand versetzen, sondern tatsächlich auch die Neubildung von Blutgefäßen verhindern kann.

Literatur:

Stoll SJ, Bartsch S, Augustin HG et al (2011) The transcription factor HOXC9 regulates endothelial cell quiescence and vascular morphogenesis in zebrafish via inhibition of interleukin 8. Circulation Research 108:1367-1377

Quelle: Medizinische Fakultät Mannheim der Universität Heidelberg, www.umm.de 